\title{
Power Allocation for Opportunistic Full-Duplex based Relay Selection in Cooperative Systems
}

\author{
Bin Zhong ${ }^{1,2}$, Dandan Zhang ${ }^{2}$ and Zhongshan Zhang ${ }^{2}$, \\ ${ }^{1}$ School of Information and Electrical Engineering, Hunan University of Science and Technology \\ Xiangtan, Hunan Province, 411201 -China \\ [e-mail: zhongbin-1982@163.com] \\ ${ }^{2}$ Beijing Engineering and \\ Technology Research Center for Convergence Networks and Ubiquitous Services, \\ University of Science and Technology Beijing (USTB) \\ Beijing, 100083 -China \\ [e-mail: zhangdandan0310@163.com,zhangzs@ustb.edu.cn] \\ *Corresponding author: Zhongshan Zhang
}

Received March 15, 2015; revised June 28, 2015; revised August 2, 2015; accepted August 23, 2015; published October 31, 2015

\begin{abstract}
In this paper, performance analysis of full-duplex (FD) relay selection under decode-and-forward (DF) relaying mode is carried out by taking into account several critical factors, including the distributions of the received signal-to-noise ratio (SNR) and the outage probability of wireless links. The tradeoff between the FD and half-duplex (HD) modes for relay selection techniques is also analyzed, where the former suffers from the impact of residual self-interference, but the latter requires more channel resources than the former (i.e., two orthogonal channels are required). Furthermore, the impact of optimal power allocation (OPA) on the proposed relay-selection scheme is analyzed. Particularly, the exact closed-form expressions for outage probability of the proposed scheme over Rayleigh fading channels are derived, followed by validating the proposed analysis using simulation. Numerical results show that the proposed FD based scheme outperforms the HD based scheme by more than 4 $\mathrm{dB}$ in terms of coding gain, provided that the residual self-interference level in the FD mode can be substantially suppressed to the level that is below the noise power.
\end{abstract}

Keywords: Decode-and-forward, full-duplex, outage probability, power allocation, relay selection

This work was supported by the National Natural Science Foundation of China (Grant No.61501182), the 863 project No. 2014AA01A701, Program for New Century Excellent Talents in University (NECT-12-0774), the Research Foundation of Education Department of Hunan Province,China (Grant No. 15C0558), the Open Research Fund of National Mobile Communications Research Laboratory Southeast University (No.2013D12), Fundamental Research Funds for the Central Universities, and the Foundation of Beijing Engineering and Technology Center for Convergence Networks and Ubiquitous Services. 


\section{Introduction}

Since modern communication networks must deliver an ever increasing data rate in order to satisfy the users' traffic demand, the spectral efficiency (SE, as measured in bits $/ \mathrm{s} / \mathrm{Hz} / \mathrm{km}^{2}$ ) of the wireless networks should be substantially improved. Apart from it, the radio coverage should also be extended for facilitating a seamless wireless access to the mobile users. Cooperative communications system comprising multiple relays, regarded as a promising solution for combating the shadowing effect, extending the radio coverage and improving the network throughput, facilitating a better immunity against signal fading and a more system-wide power saving, etc., has attracted a wide attention [1]-[4].

However, the spectrum-utilization penalty in classical resource-allocation policies may even deteriorate the benefits from using multiple relays, if an orthogonal channel allocation (e.g., in terms of carrier frequencies, time slots or codes) among relays is required. In order to address the above-mentioned problem, opportunistic relay selection relying on limited channel state information (CSI) feedback can be implemented [5]-[9]. The existed studies showed that the method of forwarding the source's data via the optimally chosen relay is effective in balancing the achievable diversity order and the attainable SE [10]-[12].

Many of the existing studies have been focused on half-duplex (HD) relaying mode [13], [14]. Unlike the HD mode, the full-duplex (FD) relaying mode allows for concurrent transmission and reception of a communication device in a single time/frequency channel [15]-[18], thus substantially improving the system's SE. However, as a downside, the FD mode may suffer from a performance erosion imposed by the self-interference ${ }^{1}$. A substantial SE-improvement than the conventional HD mode can be attained [19], [20] as long as the self-interference in the FD devices can be effectively suppressed.

Apart from it, power allocation would also play an important role in effectively suppressing the self-interference power imposed on the FD devices [21], [22]. Several works have been performed to deal with the power allocation in FD relays (see, e.g. [23] and the references therein). Generally, power allocation techniques in single-relay systems can be developed subject to one of the following two constraints:

- The individual power constraints (IPC), in which the individual power of both the source $(S)$ and relay $(R)$ should be determined by the control unit (CU);

- The sum power constraints (SPC), in which only the sum power of $S$ and $R$ is necessarily controlled by the CU.

Again, the spatial diversity gain can be substantially improved by employing multiple FD relays [24]. However, power allocation for FD based multiple-relay-selection algorithms is still left for further study.

In this paper, opportunistic decode-and-forward (DF) based FD relay selection with power allocation in cooperative communications systems is studied. The main contributions of this paper are emphasized as follows:

1) The outage probability of the wireless links under FD relay selection scheme is studied;

\footnotetext{
${ }^{1}$ Self-interference in FD systems originates from the large power difference between the device's own wireless transmissions and the received signal of interest coming from a remote transmitting antenna [15].
} 
2) The closed-form expression for the outage probability of the proposed scheme over independent and identically distributed (i.i.d.) Rayleigh fading channels is derived;

3) The proposed theoretical analysis is validated by using simulations;

4) The spatial diversity order of the proposed FD relay selection scheme is shown to be independent of the variance of residual self-interference-to-noise ratio in the FD relay devices.

The remainder of this paper is organized as follows. Section 2 introduces the system model of opportunistic FD relay selection. Both the cumulative distribution function (CDF) and probability density function (PDF) of the received signal-to-noise ratio (SNR) at the destination are also derived in this section, followed by the closed-form expressions of outage probability subject to various power allocation policies, such as equal power allocation (EPA), optimal power allocation (OPA) under IPC and SPC, in Section 3. Section 4 gives out the numerical results. Finally, Section 5 concludes this paper.

Notation: $F_{X}(\cdot)$ and $f_{X}(\cdot)$ represent the CDF and PDF of the random variable (RV) $X$, respectively. $\operatorname{Var}(\mathrm{X})$ represents the varance of the RV $X$.

\section{System Model}

In this section, we consider a cooperative network comprising a source node $S, N$ parallel FD relays operating at DF mode, and a destination $D$ (please see Fig. 1). Note that the FD-based relay receives and transmits data simultaneously via the same frequency band, the residual self-interference (i.e., $\gamma_{L I}$ ) will always be non-zero even after performing self-interference cancellation ${ }^{2}$. Without loss of generality, the direct $S \rightarrow D$ link is assumed to suffer from deep fading and will be unavailable for signal transmission. Furthermore, we assume that $S$ and $R_{i}$ perform signal transmission/forward with power of $P_{S}$ and $P_{R}$, respectively. Additionally, each node receives and transmits data with single antenna, respectively, and suffers from additive white Gaussian noise (AWGN) with zero mean and variance $\sigma_{0}^{2}$.

In the following, we consider the DF rather than AF relaying mode to perform signal forwarding. The signal is transmitted with one phase according to the essential of FD relaying mode. Furthermore, assume $S$ transmits $x(\mathrm{t})$ at time $t$, the received signals for $R_{i}$ and $D$ can be given by

$$
y_{R_{i}}(\mathrm{t})=\sqrt{P_{S}} h_{S R_{i}} x(\mathrm{t})+\sqrt{P_{R}} h_{R_{i} R_{i}} x(\mathrm{t}-\tau)+\mathrm{n}_{R_{i}}(\mathrm{t})
$$

and

$$
y_{D}(\mathrm{t})=\sqrt{P_{R}} h_{R_{i}} x(\mathrm{t}-\tau)+\mathrm{n}_{D}(\mathrm{t}),
$$

respectively, where $h_{a b}$ denotes the circularly symmetric complex Gaussian channel gain, $a, b \in\left\{R_{i}, S, D\right\}, \mathrm{n}_{R_{i}}(\mathrm{t})$ and $\mathrm{n}_{D}(\mathrm{t})$ represent the AWGN received at $R_{i}$ and $D$, respectively, and $\tau$ denotes the signal-processing time delay for FD relays.

Therefore, the PDF of SNR for $a \rightarrow b$ link can be derived as $f_{\gamma_{a b}}(\gamma)=1 /\left(P_{a} \bar{\gamma}_{a b}\right) \cdot e^{-\gamma /\left(P_{a} \bar{\gamma}_{a b}\right)}$, where $\bar{\gamma}_{a b}=\operatorname{Var}\left(h_{a b}\right) / \sigma_{0}^{2}$ and $\gamma_{a b}=\left|h_{a b}\right|^{2} / \sigma_{0}^{2}$ denote the average SNR and instantaneous SNR of

\footnotetext{
${ }^{2}$ Some typical techniques such as passive suppression, active analog and digital cancellations can be employed [19].
} 
the $a \rightarrow b$ link, respectively, where $a, b \in\{S, D\} \cup \Phi$ and $\Phi=\left\{R_{1}, R_{2}, \ldots ., \mathrm{R}_{N}\right\}$, with $h_{a b}$ denoting the circularly symmetric complex Gaussian channel gain.

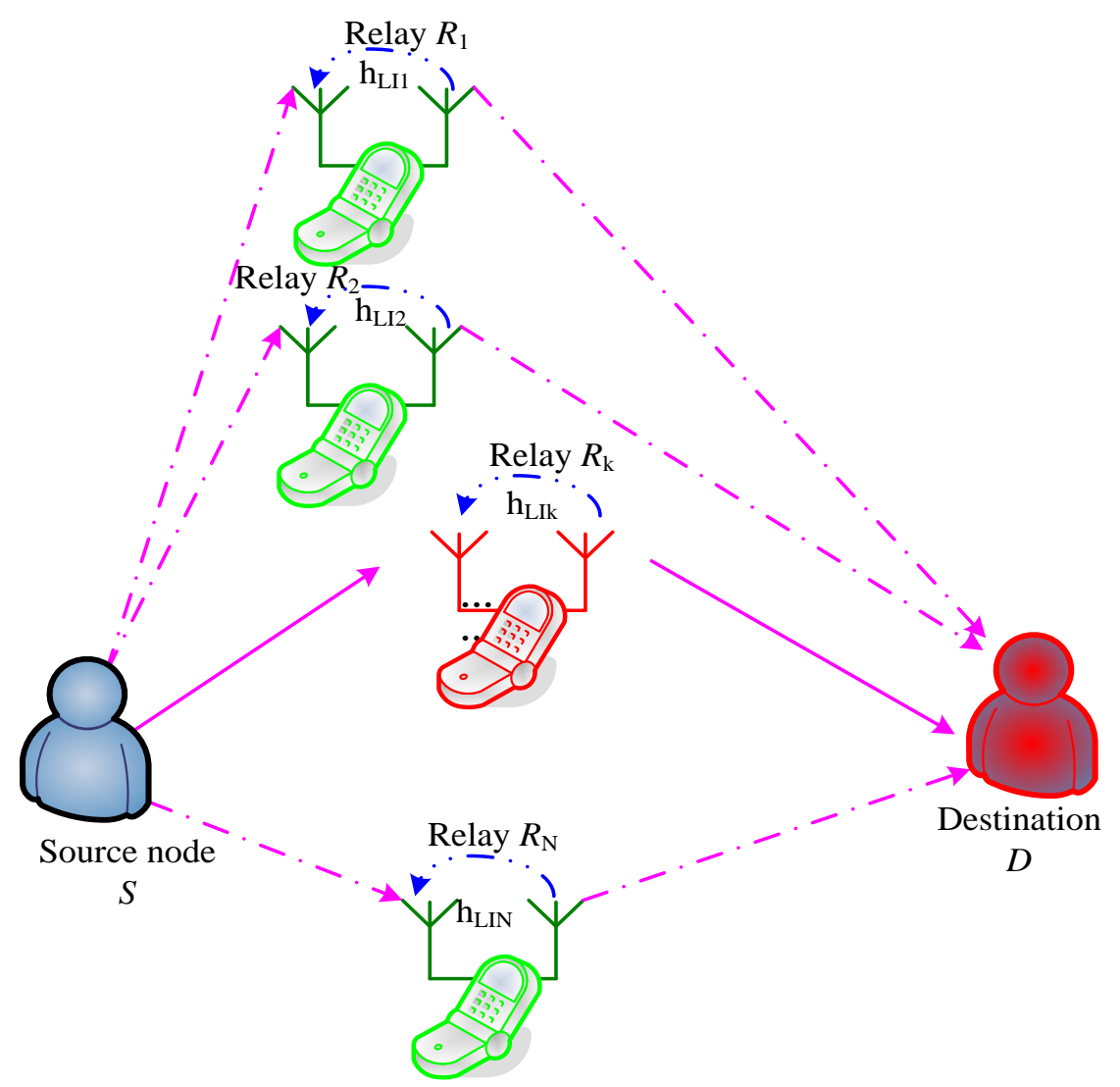

Fig. 1. A cooperative network comprising multiple FD relays.

The max-min relay selection scheme activates the relay having the best end-to-end link, while considering the impact of the residual self-interference, as given by [23, Eq. 3]

$$
k=\arg \max _{i: R_{i} \in \Phi} \min \left\{\gamma_{R_{i}}, \gamma_{D}\right\},
$$

where $\gamma_{D}$ denotes the received SNR of $D$, and $\gamma_{R_{i}}$ represents the received SNR of the $i$-th relay. By taking into account the impact of self-interference at the FD relays, the equivalent received SNR at $R_{i}$ and $D$ can be formulated as [25]

$$
\left\{\begin{array}{l}
\gamma_{R_{i}}=\frac{P_{S} \gamma_{S R_{i}}}{P_{R} \gamma_{L I}+1}, \\
\gamma_{D}=P_{R} \gamma_{R_{i} D},
\end{array}\right.
$$

where $\gamma_{L I}$ denotes the residual self-interference-to-noise ratio at the relay, and $\gamma_{R_{i} D}=\left|h_{R_{i} D}\right|^{2} / \sigma_{0}^{2}$ denotes the channel power to noise ratio at each $R_{i} \rightarrow D$ link. Consequently, the equivalent SNR of the activated link can be represented as $\gamma_{e q}=\min \left\{\gamma_{R_{k}}, P_{R} \gamma_{R_{k} D}\right\}$. 
The above-mentioned relay selection method is assumed to be controlled by a CU device, which collects all the information regarding the instantaneous CSI and then feeds back the link-selection decision to the relays. The CU thus activates the relay that satisfies (1). Hence, the CU requires the CSI knowledge of all the $\forall S \rightarrow R_{i}$ and $R_{i} \rightarrow D$ channels as well as self-interference level. It is worth mentioning that the CU based control requires an additional power consumption and bandwidth reservation. Without loss of generality, the backhaul cost of CU is neglected in this paper. Furthermore, the self-interference power at the relays can be substantially reduced by employing some existed cancellation techniques operating at both the analog and digital domains, making the residual self-interference channels $\left(h_{L I}\right)$ be modelled as Rayleigh fading parameters with a reasonable validity.

In order to evaluate the link quality of the proposed systems, both the PDF and CDF of the equivalent SNR should be derived. From Appendix I (25), the CDF of $\gamma_{e q}$ can be derived as

$$
F_{\gamma_{e q}}(x)=[\mathrm{D}(x)]^{N},
$$

where $\mathrm{D}(x)=1-\frac{P_{S} \bar{\gamma}_{S R}}{P_{S} \bar{\gamma}_{S R}+P_{R} \bar{\gamma}_{L I} x} \times \exp \left[-\left(\frac{1}{P_{R} \bar{\gamma}_{R D}}+\frac{1}{P_{S} \bar{\gamma}_{S R}}\right) \cdot x\right], \bar{\gamma}_{L I}$ denotes the variance of residual self-interference-to-noise ratio, $\bar{\gamma}_{S R}$ and $\bar{\gamma}_{R D}$ represent the average SNR of $S \rightarrow R_{k}$ and $R_{k} \rightarrow D$ link, respectively. Furthermore, the PDF of the received SNR can be derived as

$$
f_{\gamma_{e q}}(x)=\frac{N P_{S} \bar{\gamma}_{S R} \cdot[\mathrm{D}(x)]^{N-1}}{P_{S} \bar{\gamma}_{S R}+P_{R} \bar{\gamma}_{L I} X} \cdot \exp \left\{-\left(\frac{1}{P_{R} \bar{\gamma}_{R D}}+\frac{1}{P_{S} \bar{\gamma}_{S R}}\right) x\right\} \cdot\left(\frac{P_{R} \bar{\gamma}_{L I}}{P_{S} \bar{\gamma}_{S R}+P_{R} \bar{\gamma}_{L I} X}+\frac{1}{P_{R} \bar{\gamma}_{R D}}+\frac{1}{P_{S} \bar{\gamma}_{S R}}\right) .
$$

As compared to the HD relay selection schemes, the FD relay selection schemes cannot be optimized by simply increasing the transmit power of relays (i.e., for improving the received SNR of $R_{i} \rightarrow D$ link) owing to the performance erosion imposed by the enhanced residual self-interference power. Therefore, performing a proper power control method would play a critical role in optimizing the FD based opportunistic relay-selection systems.

\section{Outage Probability Analysis}

In this section, the closed-form expressions of the outage probability for both FD (with EPA and OPA) and HD modes are derived. Without loss of generality, normalized transmission power is assumed at both the source and relay nodes, whereas the total transmission power is normalized to 2 units.

\subsection{Outage Probability Subject to EPA Rule}

From (5), for a given pre-set threshold ${ }^{3} \gamma_{t h}$, the outage probability of the opportunistic relay selection scheme can be derived as

$$
P_{\text {out }}=\left[\mathrm{D}\left(\gamma_{\mathrm{th}}\right)\right]^{N},
$$

\footnotetext{
${ }^{3}$ Note that the threshold $\gamma_{t h}$ is associated with the target data rate $\alpha$. When the relays work in the FD mode, we have $\gamma_{t h}=2^{\alpha}-1$. In the HD mode, on the other hand, we have $\gamma_{t h}=2^{2 \alpha}-1$.
} 
where we have assumed that all the activated nodes transmit signals at their respective maximum power level. The optimization problem is a convex-optimization problem in terms of $\left(P_{S}, P_{R}\right)$. Furthermore, The optimum power for outage probability can be solved by using sub-gradient method.

\subsection{Outage Probability Subject to OPA Rule}

In this mode, outage probability can be formulated as

Note that (8) can be further simplified as

$$
\left(P_{S}^{*}, P_{R}^{*}\right)=\arg \min _{\left(P_{S}, P_{R}\right)} P_{\text {out }} .
$$

$$
\left(P_{S}^{*}, P_{R}^{*}\right)=\arg \max _{\left(P_{S}, P_{R}\right)} f\left(P_{S}, P_{R}\right),
$$

where $\quad f\left(P_{S}, P_{R}\right)=\frac{1}{1+a \frac{P_{R}}{P_{S}}} \exp \left\{-\left(\frac{b}{P_{R}}+\frac{c}{P_{S}}\right)\right\}$, with $a=\frac{\gamma_{t h} \bar{\gamma}_{L I}}{\bar{\gamma}_{S R}}, b=\frac{\gamma_{t h}}{\bar{\gamma}_{R D}}$, and $c=\frac{\gamma_{t h}}{\bar{\gamma}_{S R}}$.

Evidently, (9) is a monotonically increasing function of $P_{S}$. Furthermore, the first factor of $f\left(P_{S}, P_{R}\right)$, i.e. $1 /\left(1+a \frac{P_{R}}{P_{S}}\right)$ (the second factor of $f\left(P_{S}, P_{R}\right)$, i.e. $\left.\exp \left\{-\left(\frac{b}{P_{R}}+\frac{c}{P_{S}}\right)\right\}\right)$ is a monotonically deceasing (increasing) function of $P_{R}$. The outage probability can thus be minimized by using the OPA rule under policies of IPC and SPC, respectively.

1) Outage Probability under IPC Policy: The OPA based outage probability under IPC policy can be formulated as

$$
\begin{aligned}
& \left(P_{S}^{*}, P_{R}^{*}\right)=\arg \max _{\left(P_{S}, P_{R}\right)} f\left(P_{S}, P_{R}\right), \\
& \text { subject to: } 0 \leq\left\{\mathrm{P}_{S}, \mathrm{P}_{\mathrm{R}}\right\} \leq 1 .
\end{aligned}
$$

Furthermore, (10) is not jointly convex subject to $P_{S}$ and $P_{R}$. Hence, the optimal power allocation of the source and relay (OPA under IPC) can thus be derived as

$$
\left\{\begin{array}{l}
\frac{\partial f\left(P_{S}, P_{R}\right)}{\partial P_{R}}=0, \\
P_{S}^{*}=1, \\
0 \leq\left\{P_{S}^{*}, P_{R}^{*}\right\} \leq 1,
\end{array}\right.
$$

leading to

$$
\left\{\begin{array}{l}
P_{R}^{*}=\min \left\{1, \frac{a b+\sqrt{(a b)^{2}+4 a b}}{2 a}\right\} . \\
P_{S}^{*}=1 .
\end{array}\right.
$$

When $\bar{\gamma}_{L I}=0$, (12) reduces to $P_{R}^{*}=P_{S}^{*}=1$, corresponding to the HD mode.

2) Outage Probability under SPC Policy: In this case, the OPA under SPC policy can be formulated as

$$
\begin{aligned}
& \left(P_{S}^{*}, P_{R}^{*}\right)=\arg \max _{\left(P_{S}, P_{R}\right)} f\left(P_{S}, P_{R}\right) \\
& \text { subjectto: }\left\{\begin{array}{l}
\mathrm{P}_{\mathrm{S}}+\mathrm{P}_{\mathrm{R}}=2, \\
\left\{\mathrm{P}_{\mathrm{S}}, \mathrm{P}_{\mathrm{R}}\right\} \geq 0 .
\end{array}\right.
\end{aligned}
$$


The optimal transmit power allocation can thus be achieved by solving

$$
\left\{\begin{array}{l}
\frac{\partial f\left(P_{S}, P_{R}\right)}{\partial P_{R}}+\lambda=0, \\
\frac{\partial f\left(P_{S}, P_{R}\right)}{\partial P_{S}}+\lambda=0, \\
P_{S}+P_{R}=2, \\
\left\{P_{S}, P_{R}\right\} \geq 0,
\end{array}\right.
$$

with $\lambda$ denoting the Lagrangian multiplier associated under the SPC policy.

After Simplifying (14), we obtain

$$
\left\{\begin{array}{l}
\zeta P_{R}^{3}-\eta P_{R}^{2}-\varepsilon P_{R}+8 b=0, \\
P_{S}=2-P_{R}, \\
\left\{P_{S}, P_{R}\right\} \geq 0,
\end{array}\right.
$$

where $\zeta=2 a-b+c+a b-a c, \eta=4 a-6 b+2 c+4 a b$, and $\varepsilon=12 b-4 a b$.

By solving the root of the cubic polynomial of (15) using some mathematical software (e.g., Mathmatic or Matlab), we can obtain three root of $P_{R}$ as

$$
\left\{\begin{array}{l}
\omega_{1}=\frac{\eta}{3 \zeta}+\kappa+\frac{\xi}{\kappa} \\
\omega_{2}=\frac{\eta}{3 \zeta}-\frac{1-\sqrt[3]{3} \text { 㼼 }}{2}-\frac{1+\sqrt{3}}{2} \frac{\xi}{\kappa} \\
\omega_{3}=\frac{\eta}{3 \zeta}-\frac{1+\sqrt[3]{3} \text { 㼼 }}{2}-\frac{1-\sqrt{3}}{2} \frac{\xi}{\kappa}
\end{array}\right.
$$

where $\kappa=\sqrt[3]{\left(\mathrm{H}^{2}-\xi^{3}\right)^{1 / 2}+\mathrm{H}}, \mathrm{H}=\frac{\eta^{3}}{27 \zeta^{3}}-\frac{4 b}{\zeta}+\frac{\varepsilon \eta}{6 \zeta^{2}}$, and $\xi=\frac{\eta^{2}}{9 \zeta^{2}}+\frac{\varepsilon}{3 \zeta}$. When

$\exists 0 \leq \omega_{i} \leq 2, i \in\{1,2,3\}$, the optimal transmit power of relay can be derived as

$$
\left\{\begin{array}{l}
P_{R}^{*}=\omega_{i}, \\
P_{S}^{*}=2-P_{R}^{*},
\end{array}\right.
$$

When $\bar{\gamma}_{L I}=0$ and $\bar{\gamma}_{S R}=\bar{\gamma}_{R D}$, the power of $R_{k}$ and $S$ can be given by $P_{R}^{*}=1$ and $P_{S}^{*}=1$, respectively.

\section{Numerical Results}

In this section, we evaluate the proposed scheme in terms of the outage probability over i.i.d. Rayleigh fading channels by using Monte Carlo simulation. After performing self-interference cancellation at the FD relays, the residual self-interference power $\left(\sigma_{R R}^{2}\right)$ will be proportional to the power of $R_{k} \rightarrow D$ link ( $\left.\sigma_{R D}^{2}\right)$.

In Fig. 2, outage probability is evaluated by considering various number of relays (i.e. $N$ ), $\alpha=2 \mathrm{bps} / \mathrm{Hz}, \bar{\gamma}_{S R}=\bar{\gamma}_{R D}$ and $\sigma_{R R}^{2}=0.05 \sigma_{R D}^{2}$. When $N=1$ is considered (i.e., without performing relay selection), the lowest spatial diversity order is obtained. Particularly, the spatial diversity order in the FD based relaying systems can be improved by employing more relays. 


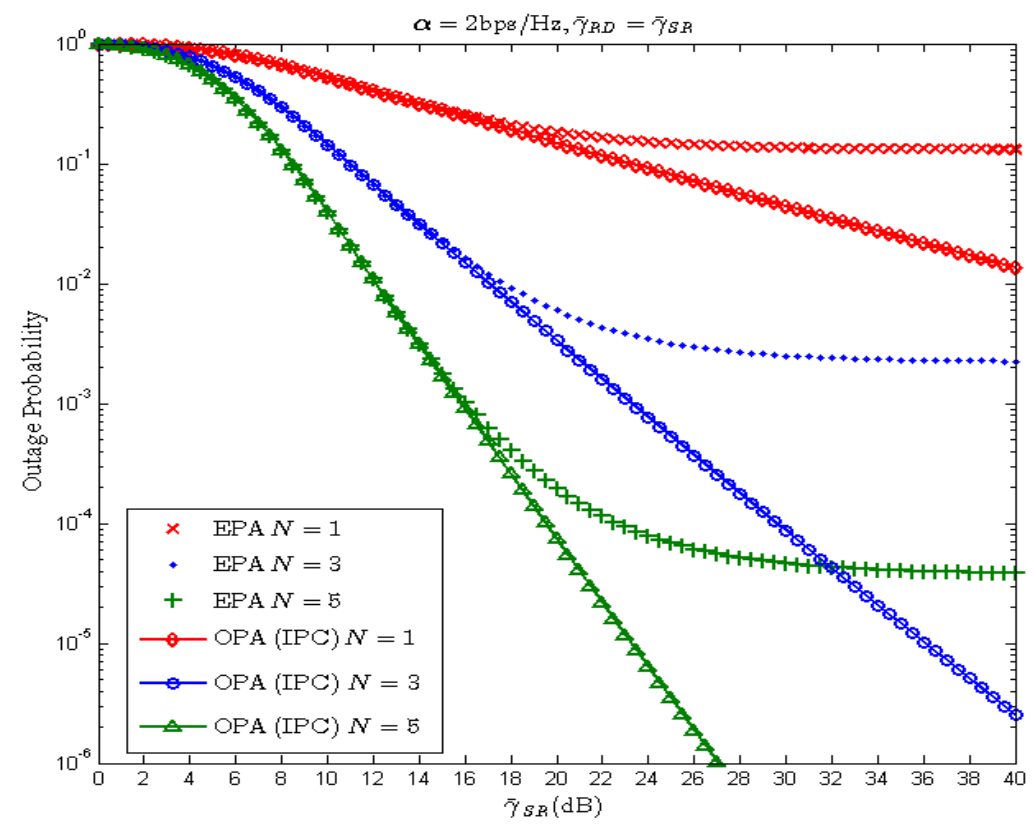

Fig. 2. Outage probability for the FD scheme with EPA and the OPA under IPC versus the average SNR of the $S \rightarrow R$ links for different $N$ with $\sigma_{R R}^{2}=0.05 \sigma_{R D}^{2}$.

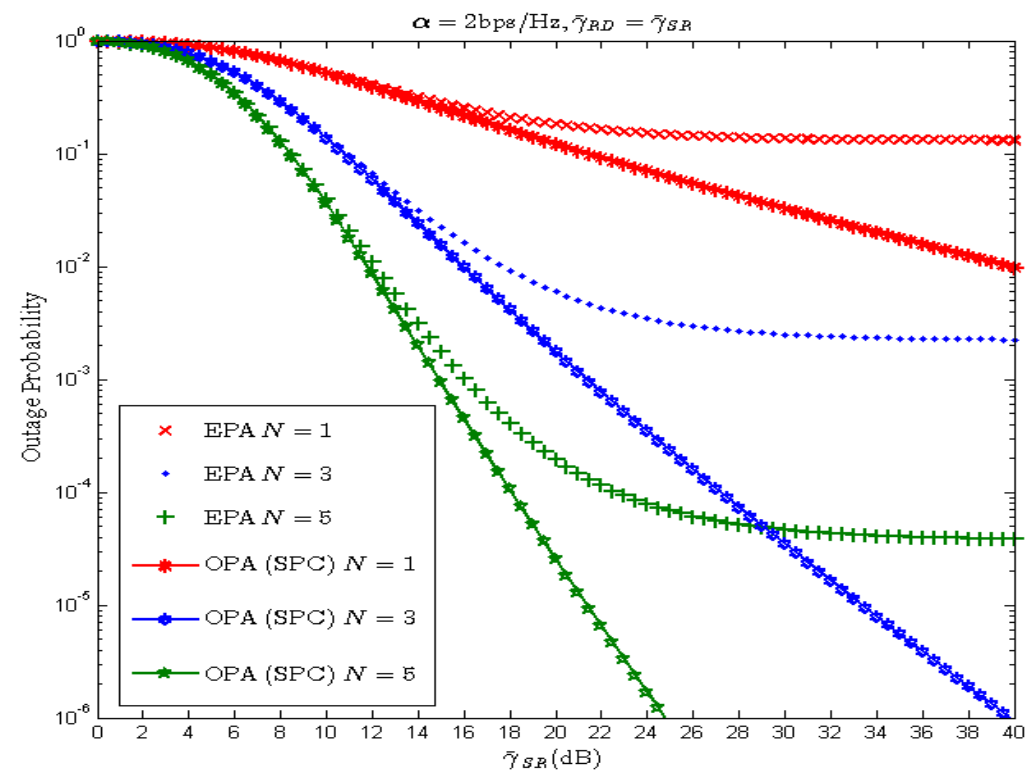

Fig. 3. Outage probability for the FD scheme with EPA and the OPA under SPC versus the average SNR of the $S \rightarrow R$ links for different $N$ with $\sigma_{R R}^{2}=0.05 \sigma_{R D}^{2}$.

As shown in Fig. 3, in the FD mode, OPA scheme under SPC policy outperforms that under IPC policy in terms of coding gain by about $2 \mathrm{~dB}$. As shown in Fig. 3, the EPA have appeared the floor effect by the residual interference powers and noise for outage probability at the high SNR. However, when the residual interference powers had been suppressed by power 
allocation, the OPA hasn't appeared the floor effect for outage probability at the high SNR. Besides, the outage performance of OPA scheme is superior to that of EPA scheme.

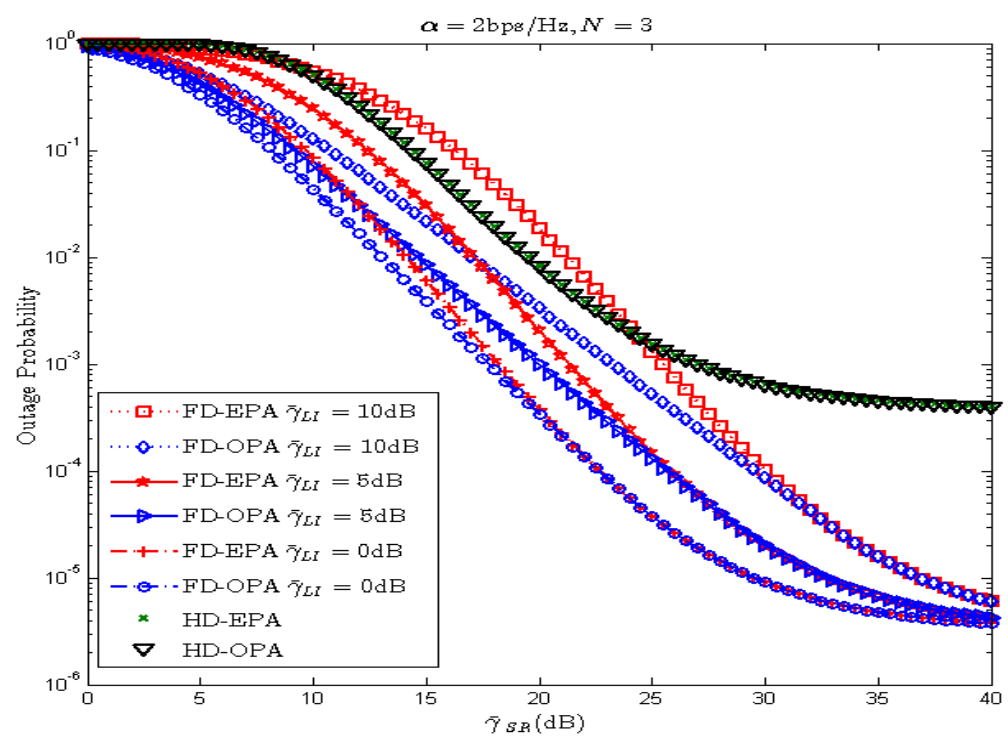

Fig. 4. Outage probability for the FD scheme with EPA, the OPA under IPC and the HD mode versus the average SNR of the $S \rightarrow R$ links for different $\bar{\gamma}_{L I}$ with $\bar{\gamma}_{R D}=23 \mathrm{~dB}$.

Fig. 4 analyzes the outage probability with $\bar{\gamma}_{R D}=23 \mathrm{~dB}$ for different $\bar{\gamma}_{L I}$ values, while keeping $N=3$ unchanged. It is shown that the curve of HD under OPA policy is identical to that under EPA policy. Meanwhile, it is also shown that the spatial diversity order in the FD relay cooperative systems becomes independent of $\bar{\gamma}_{L I}$, since the slopes of curves keep unchanged for different $\bar{\gamma}_{L I}$. If $\bar{\gamma}_{L I}<10 \mathrm{~dB}$ is satisfied, the FD mode outperforms the HD mode in terms of outage probability. Otherwise, the HD mode may obtain an advantage over the FD mode. Furthermore, it is also shown that the optimal power allocation in FD schemes under IPC policies outperform the HD mode in terms of coding gain by more than $4 \mathrm{~dB}$, provided that the self-interference can be successfully suppressed to the level that is below the noise power. The floor effect for outage probability is observed in the DF-mode cooperative communications systems. As shown in Fig. 4, increasing $\bar{\gamma}_{S R}$ implies obtaining a lower outage probability at low-SNR regime. However, if we further increase the SNR (when $\bar{\gamma}_{S R}$ approaches $25 \mathrm{~dB}$ in this simulation), the outage probability cannot be further decreased, because the selected relay has successfully decoded the symbol at the high-SNR link, and further increasing SNR may not contribute to the reduction of outage probability. Consequently, a floor effect appears at the outage probability.

\section{Conclusion}

The performance of the FD based relay selection scheme under DF relaying mode was evaluated by deriving the closed-form expressions of the CDF, the PDF and the outage probability of the activated link. The proposed scheme with OPA scheme (under IPC and SPC policies) was also validated by using simulations. Particularly, the theoretical analysis was shown to match the corresponding numerical results well. Furthermore, it was also shown in 
the numerical results that the other parameters, including the number of relays, the residual self-interference, and the SNR of relaying links, etc., all substantially impact the performance of the multi-relay systems. Finally, simulation results showed that the FD based mode could outperform the HD based mode in terms of coding gain by more than $4 \mathrm{~dB}$, provided that the self-interference at the FD relays can be sufficiently suppressed.

\section{Appendix I}

\section{The probability density function of $\gamma_{e q}$}

For a Rayleigh fading channel, the PDF of $\gamma_{S R_{i}}$ is given by

$$
f_{\gamma_{S S_{i}}}(x)=\frac{1}{\bar{\gamma}_{S R}} e^{-\frac{x}{\bar{\gamma}_{S R}}}, x \geq 0,
$$

leading to the CDF of $\gamma_{S R_{i}}$

$$
F_{\gamma_{S R_{i}}}(x)=1-e^{-\frac{x}{\bar{\gamma}_{S R}}}, \quad x \geq 0 .
$$

Similarly, the PDF of $\gamma_{L I}$ can be given by

$$
f_{\gamma_{L I}}(x)=\frac{1}{\bar{\gamma}_{L I}} e^{-\frac{x}{\bar{\gamma}_{L I}}}, \quad x \geq 0,
$$

where $\bar{\gamma}_{L I}$ denotes the average residual self-interference power.

The CDF of $\gamma_{R_{i}}$ can be derived as

$$
\begin{aligned}
F_{\gamma_{R_{i}}}(x) & =\operatorname{Pr}\left\{\gamma_{R_{i}}<x\right\} \\
& =\operatorname{Pr}\left\{\frac{P_{S} \gamma_{S R_{i}}}{P_{R} \gamma_{L I}+1}<x\right\} \\
& =\int_{0}^{\infty} \operatorname{Pr}\left\{\frac{P_{S} \gamma_{S R_{i}}}{P_{R} y+1}<x\right\} f_{\gamma_{L I}}(y) d y \\
& =\int_{0}^{\infty} \operatorname{Pr}\left\{\gamma_{S R_{i}}<\frac{\left(P_{R} y+1\right) x}{P_{S}}\right\} f_{\gamma_{L I}}(y) d y \\
& =\int_{0}^{\infty} F_{S R_{i}}\left(\frac{\left(P_{R} y+1\right) x}{P_{S}}\right) f_{\gamma_{L I}}(y) d y \\
& =\int_{0}^{\infty}\left\{1-\exp \left(-\frac{\left(P_{R} y+1\right) x}{P_{S} \bar{\gamma}_{S R}}\right)\right\} \frac{1}{\bar{\gamma}_{L I}} e^{-\frac{y}{\bar{\gamma}_{L I}}} d y \\
& =\int_{0}^{\infty}\left\{1-\exp \left(-\frac{\left(P_{R} y+1\right) x}{P_{S} \bar{\gamma}_{S R}}\right)\right\} \frac{1}{\bar{\gamma}_{L I}} e^{-\frac{y}{\bar{\gamma}_{L I}}} d y \\
& =1-\frac{P_{S} \bar{\gamma}_{S R}}{P_{S} \bar{\gamma}_{S R}+P_{R} \bar{\gamma}_{L I} x} \exp \left(-\frac{x}{P_{S} \bar{\gamma}_{S R}}\right) .
\end{aligned}
$$

Taking partial derive to (21) with respect to $x$, we can obtain the PDF of RV $\gamma_{R_{i}}$ as follows 


$$
f_{\gamma_{R_{i}}}(x)=\frac{\exp \left(-\frac{x}{P_{S} \bar{\gamma}_{S R}}\right)}{P_{S} \bar{\gamma}_{S R}+P_{R} \bar{\gamma}_{L I} x}\left(1+\frac{P_{R} \bar{\gamma}_{L I} P_{S} \bar{\gamma}_{S R}}{P_{S} \bar{\gamma}_{S R}+P_{R} \bar{\gamma}_{L I} x}\right) .
$$

where $x \geq 0$.

Furthermore, by denoting $z=\min \left\{\gamma_{R_{i}}, \gamma_{D}\right\}$, the CDF of $Z$ can be derived as

$$
F_{Z}(x)=1-\left[1-F_{\gamma_{R_{i}}}(x)\right] \cdot\left[1-F_{\gamma_{D}}(x)\right], x \geq 0,
$$

with $F_{\gamma_{D}}(x)=1-\exp \left\{-\frac{x}{P_{R} \bar{\gamma}_{R D}}\right\}$. The CDF of $Z$ can be expressed as

leading to

$$
F_{Z}(x)=1-\frac{P_{S} \bar{\gamma}_{S R}}{P_{S} \bar{\gamma}_{S R}+P_{R} \bar{\gamma}_{L I} X} \cdot \exp \left\{-\left(\frac{1}{P_{R} \bar{\gamma}_{R D}}+\frac{1}{P_{S} \bar{\gamma}_{S R}}\right) \cdot x\right\}, x \geq 0
$$

$$
F_{\gamma_{e q}}(x)=\left\{F_{Z}(x)\right\}^{N}, x \geq 0
$$

\section{References}

[1] A. Sendonaris, E. Erkip, and B. Aazhang, "User cooperation diversity-Part I: system description,” IEEE Trans. Commun., vol. 51, no. 11, pp. 1927 - 1938, Nov. 2003. Article (CrossRef Link)

[2] Z. Zhang, K. Long, and J. Wang, "Self-organization paradigms and optimization approaches for cognitive radio technologies: a survey,” IEEE Wireless Commun., vol. 20, no. 2, pp. 36-42, Apr. 2013. Article (CrossRef Link)

[3] Z. Zhang, K. Long, J. Wang, and F. Dressler, "On swarm intelligence inspired self-organized networking: its bionic mechanisms, designing principles and optimization approaches," IEEE Commun. Surv. Tut, vol. 16, no. 1, pp.513-537, 2014. Article (CrossRef Link)

[4] C. Xing, S. Ma, and Y.-C. Wu, "Robust joint design of linear relay precoder and destination equalizer for dual-hop amplify-and-forward MIMO relay systems," IEEE Transactions on Signal Processing, vol. 58, no. 4, pp. 2273-2283, Apr. 2010. Article (CrossRef Link)

[5] S. S. Ikki and M. H. Ahmed, "Performance analysis of adaptive decode-and-forward cooperative diversity networks with best-relay selection,” IEEE Trans. Commun., vol. 58, no. 1, pp. 68-72, Jan. 2010. Article (CrossRef Link)

[6] Z. Zhang, C. Tellambura, and R. Schober, "Improved OFDMA uplink transmission via cooperative relaying in the presence of frequency offsets-part I: ergodic information rate analysis,” Euro. Trans. Telecommun., vol. 21, no. 3, pp. 224-240, Apr. 2010.

Article (CrossRef Link)

[7] A. Muller and J. Speidel, "Relay selection in dual-hop transmission systems: Selection strategies and performance results," in Proc. of IEEE Int. Conf. Communications (ICC), pp. 4998-5003, 2008. Article (CrossRef Link)

[8] A. Ikhlef, J. Kim, and R. Schober, "Mimicking full-duplex relaying using half-duplex relays with buffers,” IEEE Trans. Veh. Technol., vol. 61, no. 7, pp. 3025-3037, Sept. 2012. Article (CrossRef Link)

[9] Y. Zou, Y. Yao, and B. Zheng, "Opportunistic distributed space time coding for decode-and-forward cooperation systems," IEEE Trans. Signal Processing, vol. 60, no. 4, pp. 1766-1781, Apr. 2012. Article (CrossRef Link)

[10] Z. Zhang, W. Zhang, and C. Tellambura, "OFDMA uplink frequency offset estimation via cooperative relaying," IEEE Trans. Wireless Commun., vol. 8, no. 9, pp. 4450-4456, Sept. 2009. Article (CrossRef Link) 
[11] R. U. Nabar, H. Bolcskei, and F. W. Kneubuhler, "Fading relay channels: performance limits and space-time signal design,” IEEE J. Select. Areas Commun., vol. 22, no. 6, pp. 1099-1109, Aug. 2004. Article (CrossRef Link).

[12] J. Jia, J. Zhang, and Q. Zhang, “Cooperative relay for cognitive radio network,” IEEE INFOCOM, pp. 2304-2312, 2009. Article (CrossRef Link)

[13] D. Gunduz, A. Yener, A. Goldsmith, and H. V. Poor, “The multi-way relay channel,” in Proc. of IEEE Int. Symp. on Infor.Theory (ISIT), Seoul, Korea, pp. 339-343, 2009. Article (CrossRef Link)

[14] C. Xing, S. Ma, and Y. Zhou, "Matrix-monotonic optimization for MIMO systems," IEEE Trans. Signal Processing, vol. 63, no. 2, pp. 334-348, Jan. 2015. Article (CrossRef Link)

[15] T. Riihonen, S. Werner, and R. Wichman, "Mitigation of loopback self-interference in full-duplex MIMO relays,” IEEE Trans. Signal Processing, vol. 59, no. 12, pp. 5983-5993, Dec. 2011. Article (CrossRef Link)

[16] - "Comparison of full-duplex and half-duplex modes with a fixed amplify-and-forward relay," in Proc. of IEEE WCNC, 2009. Article (CrossRef Link)

[17] T. M. Kim and A. Paulraj, "Outage probability of amplify-and-forward cooperation with full duplex relay,” in Proc. of IEEE WCNC, pp. 75-79, Apr. 2012. Article (CrossRef Link)

[18] T. Riihonen, S. Werner, and R. Wichman, "Rate-interference trade-off between duplex modes in decode-and-forward relaying,” in Proc. of IEEE PIMRC, pp. 690-695, Sept. 2010. Article (CrossRef Link)

[19] J. I. Choi, M. Jain, K. Srinivasan, P. Levis, and S. Katti, “Achieving single channel, full duplex wireless communication,” in Proc. of ACM MobiCom, pp. 1-12, Sept. 2010. Article (CrossRef Link)

[20] N. Zlatanov and R. Schober, "Buffer-aided half-duplex relaying can outperform ideal full-duplex relaying,” IEEE Commun. Lett., vol. 17, no. 3, pp. 479-482, Mar. 2013. Article (CrossRef Link)

[21] N. Nomikos, T. Charalambous, I. Krikidis, D. Vouyioukas, and M. Johansson, "Hybrid cooperation through full-duplex opportunistic relaying and max-link relay selection with transmit power adaptation,” in Proc. of IEEE ICC, pp. 5706-5711, 2014. Article (CrossRef Link)

[22] X. Rui, J. Hou, and L. Zhou, "On the performance of full-duplex relaying with relay selection," Electronics Letters, vol. 46, no. 25, pp. 1674-1676, Dec. 2010. Article (CrossRef Link)

[23] H. A. Suraweera, I. Krikidis, G. Zheng, C. Yuen, and P. J. Smith, "Low complexity end-to-end performance optimization in MIMO full-duplex relay systems,” IEEE Trans. Wireless Commun., vol. 13, no. 2, pp. 913-917, Feb. 2014. Article (CrossRef Link)

[24] I. Krikidis, H. A. Suraweera, P. J. Smith, and C. Yuen, "Full-duplex relay selection for amplify-and-forward cooperative networks,” IEEE Trans. Wireless Commun., vol. 11, no. 12, pp. 4381-4393, Dec. 2012. Article (CrossRef Link)

[25] T. Riihonen, S. Werner, R. Wichman, and E. B. Zacarias, "On the feasibility of full-duplex relaying in the presence of loop interference," in Proc. of IEEE Workshop Signal Process. Adv. Wireless Commun (SPAWC), Perugia, Italy, June 2009. Article (CrossRef Link) 


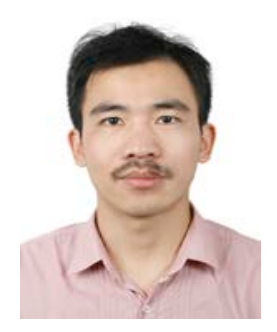

Bin Zhong received his B.Sc. degree in electronic and information engineering from Xiangtan University, Xiangtan, China in 2005, the M.Sc. degree in detection technology and automatic equipment from Guilin University of Electronic Technology, Guilin, China in 2011, the Ph.D. degree in communication and information system from University of Science and Technology Beijing (USTB), Beijing, China in 2014. He is currently a Lecturer of the school of information and electrical engineering in the Hunan University of Science and Technology, Xiangtan, China. His current research interests include wireless communications theory, cognitive networks, and diversity and cooperative communications. He is also a recipient of "Best Paper Award" at IEEE International Conference on Communication Technology, September 2012, Chengdu, China.

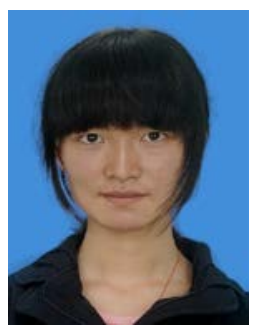

Dandan Zhang received her B.Sc. degree in communication engineering in University of Science and Technology Beijing in 2013. She is currently working toward the M.Sc. degree with the Beijing Engineering and Technology Research Center for Convergence Networks and Ubiquitous Services, University of Science and Technology Beijing (USTB), Beijing, China. Her current research interests include wireless communications theory, cognitive radio, and cooperative communications.

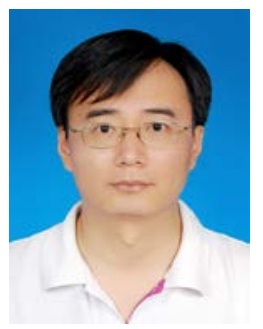

Zhongshan Zhang received the B.E. and M.S. degrees in computer science from the Beijing University of Posts and Telecommunications (BUPT) in 1998 and 2001, respectively, and received Ph.D. degree in electrical engineering in 2004 from BUPT. From Aug. 2004 he joined DoCoMo Beijing Laboratories as an associate researcher, and was promoted to be a researcher in Dec. 2005. From Feb. 2006, he joined University of Alberta, Edmonton, AB, Canada, as a postdoctoral fellow. From Apr. 2009, he joined the Department of Research and Innovation (R\&I), Alcatel-Lucent, Shanghai, as a Research Scientist. From Aug. 2010 to Jul. 2011, he worked in NEC China Laboratories, as a Senior Researcher. He is currently a professor of the School of Computer and Communication Engineering in the University of Science and Technology Beijing (USTB). His main research interests include statistical signal processing, self-organized networking, cognitive radio, and cooperative communications. 